\title{
Práctica de la iridología como herramienta diagnóstica en medicina natural
}

\section{Shirley del Carmen Pérez Gamboa}

shipere3@gmail.com

\section{Eulalia Isabel Analuisa Jiménez \\ ei.analuisa@uta.edu.ec}

Facultad de Ciencias de la Salud.

Universidad Técnica de Ambato

Ambato- Ecuador

\section{RESUMEN}

La Iridología es una seudociencia utilizada como herramienta diagnóstica que tiene a fin la prevención de posibles enfermedades futuras, así también es útil cómo método de recuperación del estado de salud del paciente; La Iridología va de la mano con la medicina natural u homeopatía, ya que, a través de la utilización de agentes naturales, se logra obtener resultados favorables sobre la salud de las personas. Objetivo: Analizar mediante una revisión bibliográfica la práctica de la Iridología como herramienta diagnóstica en medicina natural. Método: Se realizó una revisión de metaanálisis donde se ejecutó una búsqueda bibliográfica en: Google académico, SciELO, Medline, Dialnet, así como una búsqueda manual entre las referencias de los estudios seleccionados y en diferentes bibliotecas. Resultados: el artículo trata la teoría y la historia de la Iridología, las áreas que se identifican en el iris del ojo, así como las diferentes formas y coloraciones que se encuentran en el mismo. También se analizan algunos diagramas para poder llegar a un diagnóstico verídico. Conclusión: En Ecuador debemos realizar investigaciones sobre Iridología, porque no encontramos información científica sobre esto, sin embargo, la homeopatía es muy reconocida en dicho país.

Palabras clave: iridología; medicina natural; homeopatía; medicina tradicional. 


\title{
Practice of iridology as a diagnostic tool in natural medicine
}

\begin{abstract}
Iridology is a pseudo-science used as a diagnostic tool for the prevention of possible future diseases, and it is also useful as a method of recovery of the patient's state of health; Iridology goes hand in hand with natural medicine or homeopathy, since through the use of natural agents (plants, etc.) it is possible to obtain favorable results on people's health. Objective: To analyze through a bibliographic review the practice of Iridology as a diagnostic tool in natural medicine. Method: A meta-analysis review was performed where a literature search was executed in: Google academic, SciELO, Medline, Dialnet, as well as a manual search among the references of the selected studies and in different libraries. Results: The article discusses the theory and history of Iridology, the areas that are identified in the iris of the eye, as well as the different shapes and colorations found in it. Some diagrams are also analyzed in order to arrive at a true diagnosis. Conclusion: In Ecuador we should carry out research on Iridology, because we did not find scientific information about this, however, homeopathy is well recognized in that country.
\end{abstract}

Keywords: Iridology, natural medicine, homeopathy, traditional medicine.

Artículo recibido: 30 noviembre. 2021 Aceptado para publicación: 29 diciembre 2021

Correspondencia: shipere3@gmail.com Conflictos de Interés: Ninguna que declarar 


\section{INTRODUCCIÓN}

El panorama de salud en todo el planeta es alarmante. La medicina tradicional y los sistemas de salud no han logrado satisfacer las necesidades de la población; cuidando de la salud. Hoy en día, la gente busca constantemente alternativas para aliviar su dolor, sin ser demasiado caro y causar demasiados efectos secundarios.(López González, Pérez González, and Artiles López 2016)

Las principales razones que motivan a los pacientes a buscar estas terapias son: la búsqueda de la promoción de la salud y la prevención de enfermedades; porque es beneficioso tanto para la salud emocional y salud espiritual; porque la efectividad de la medicina convencional es indeterminado o generalmente asociado a efectos secundarios y riesgos. Varios profesionales de la salud están comenzando estudiar algún tipo de terapia complementaria que puede, junto con su área original, mejorar la atención brindada a su paciente.(Salles and de Silva 2018)

Según cita Luna,(Flower 2016) la Idología es un método de diagnóstico clínico basado en la observación de la morfología, la estructura y los signos que presentan los individuos en el iris del ojo, revelando así el estado de salud de un individuo; aunque la Iridología surgió a mediados del siglo XIX por su fundador el médico húngaro Ignaz von Peczely (1826-1911), siendo considerada como una pseudoterapia. Desde este comienzo del Dr. Ignaz von Peczely y sus posteriores investigaciones exhaustivas durante un periodo de cuarenta años, científicos eminentes en Alemania, Inglaterra, Suecia y América han agregado sus valiosas contribuciones de modo que la Iridología actual puede ser legítimamente considerada como una ciencia exacta. Hombres como el Dr. Liljequist, Dr. Anderschou, Dr. Thiele, Dr. Lahn, Dr. Lindlaher, Dr. Kritzer y otros, deben recibir el mérito del actual estado de perfección que ha traído el iridiagnóstico. Fue en Estados Unidos, en la década de 1950, donde el Doctor Bernard Jensen realizó un diagrama detallado del iris de ambos ojos, en el que señalaba la parte exacta del cuerpo con que estaba relacionada cada zona del iris. (Flower 2016)

Esta herramienta diagnóstica fue desarrollada hace más de 100 años asumiendo que todos los órganos corporales están representados en la superficie del iris, a través de intrincadas conexiones neurales y que la mayoría de las disfunciones de varios órganos están marcados en el iris, generalmente como un cambio de pigmentación. Sabiendo que la 
parte derecha del cuerpo está representado en el iris derecho y la parte izquierda del cuerpo la representa el iris izquierdo. (Ernst 2016)

El iris es un microsistema, completamente formado a los seis años, que permite al iridólogo realizar un abordaje profiláctico y terapéutico, a través del prediagnóstico. Una vez que se detectan las señales de peligro, estos órganos deben investigarse con exámenes específicos para eliminar la posibilidad de ciertas enfermedades.(Salles, Silva, and Araújo 2018)

La Homeopatía es un sistema de medicina alternativa creado en 1796 por Samuel Hahnemann, basado en su doctrina de lo similar cura lo similar, donde una sustancia que cause los síntomas de una enfermedad en personas sanas curará lo similar en personas enfermas. Constituye un método terapéutico que se basa en el principio de similitud entre los poderes toxicológico y terapéutico de una sustancia. (Oliveira et al. 2018) Al respecto, existen sustancias que cuando se utilizan en grandes dosis provocan enfermedades, mientras que si se emplean en cantidades infinitesimales curan síntomas análogos a los que han provocado.(López Vantour et al. 2017) Este método se caracteriza por la individualización del tratamiento; tiene en cuenta que existen enfermos, no enfermedades, por lo que dicho tratamiento dependerá siempre de la reacción del paciente. Creagh et al, citan a Nápoles González, quien refiere que actualmente la homeopatía se utiliza en más de 80 naciones, principalmente en Europa, América del Sur, India y Paquistán, aunque en América del Norte no está aún lo suficientemente expandida. A partir de 1946, cuando los doctores Francisco de Paula Escofet y Valentín Catalá la emplearon en Cuba, su uso se extendió paulatinamente a todo el país.(López González et al. 2016)

Además, la homeopatía se utiliza en la actualidad en más de 80 países, con más de 300 millones de pacientes y alrededor de 248400 médicos que la prescriben en sus consultas. En otros países de Europa, la homeopatía es una práctica habitual, y en algunos forma parte incluso de los sistemas sanitarios. Es el caso de Francia, por ejemplo, con más de 30000 médicos prescribiéndola y con un $56 \%$ de la población que utiliza la homeopatía en la actualidad, o Suiza, donde la mitad de la población ha usado homeopatía alguna vez y el $85 \%$ considera que deberían formar parte del sistema de salud pública. (Sacristán Rubio and Torres Jiménez 2016) 


\section{MÉTODOS}

Artículo de revisión bibliográfica donde se identificó el número de bases de datos y también resúmenes identificados, mediante otras fuentes, donde se utilizó cierta cantidad de artículos que tenían información relevante sobre el tema. Se realizo un tamizaje del número de resúmenes o artículos encontrados y se obtuvo un total de artículos. Se ejecuto una búsqueda bibliográfica en: Google académico, SciELO, Medline, Dialnet, así como una búsqueda manual entre las referencias de los estudios seleccionados y en diferentes bibliotecas, buscando palabras claves como: Iridología (Iridology), homeopatía, medicina natural, iris. Además de la búsqueda computadorizada se realizó una investigación manual entre las referencias de los estudios seleccionados. (Marín Martínez, Sánchez Meca, and López López 2019)

Se realizó una revisión de metaanálisis, sabiendo que ésta, es una herramienta estadística para estimar la media y la varianza de los efectos poblacionales subyacentes a partir de una colección de estudios empíricos que abordan aparentemente la misma pregunta de investigación.(Yuliet and Fandiño 2019) El metaanálisis se ha convertido en una herramienta cada vez más popular y valiosa en la investigación psicológica, y los principales artículos de revisión suelen emplear estos métodos. Este artículo describió el proceso como realizar un metaanálisis: seleccionando artículos, desarrollando criterios de inclusión, calculando los tamaños del efecto, realizando el análisis real y estimando los efectos de sesgo publicación.(Delgado Rodríguez and Palma Pérez 2016)

El metaanálisis es una metodología para la revisión sistemática y cuantitativa de la investigación, ampliamente consolidada y aplicada en las Ciencias de la Salud. Ofrece las técnicas necesarias para acumular rigurosa y eficientemente los resultados cuantitativos de los estudios empíricos sobre un mismo problema de salud, permitiendo a los profesionales de la salud la adopción de decisiones bien informadas en sus respectivas áreas de trabajo.(Ochoa Ortega 2018) Se empleo, este tipo de investigación porque permite integrar mediante modelos estadísticos apropiados los resultados obtenidos en diferentes estudios que por su lado han intentado responder el mismo problema de investigación; evaluar si existe homogeneidad estadística entre los estudios, aplicar métodos estadísticos para determinar el aporte de cada estudio al resultado final e identificar las inconsistencias (si las hay) en los resultados de investigaciones previas.(Yuliet and Fandiño 2019) 


\section{RESULTADOS Y DISCUSIÓN}

Historia: La Iridología es una ciencia que estudia los patrones estructurales y de pigmentación y otros parámetros relacionados con el iris del ojo para analizar la constitución de un individuo.(Salles and de Silva 2018) En la historia de la Iridología, según cita Esteves et al.,(Esteves et al. 2021) el húngaro Ignatz von Peczely, en 1881, publicó un libro: Descubrimientos en el campo de las ciencias naturales y la medicina: instrucción en el estudio del diagnóstico desde el ojo, que presentaba un mapa topográfico del iris que refleja áreas del cuerpo. El autor se hizo conocido como el padre de la Iridología.(Ernst 2016) Hay más de 80 áreas identificadas en los mapas topográficos del iris, que varían debido a la existencia de diferentes marcos teóricos. El cuerpo de conocimientos sobre Iridología se aplica en la práctica de la medicina complementaria e integrativa, principalmente a través de las prácticas de los terapeutas holísticos.(Flower 2016)

La Iridología, iridodiagnosis o iriscopía es la ciencia que permite a un profesional detectar enfermedades sistémicas mediante el estudio de imágenes del iris del paciente. Es un tipo de medicina complementaria, cuyos practicantes estudian los patrones, colores y otras características del iris con el fin de evaluar la salud sistémica del paciente.(Luna 2016) Los practicantes de esta ciencia comparan sus observaciones con las cartas de iridología, ya descritas por otros autores, en las que cada parte del iris corresponde con una parte específica del cuerpo.(Llergo 2020)

Al examinar el iris, se busca determinar la ubicación y la naturaleza de posibles alteraciones en el organismo y, por tanto, para detectar predisposiciones a determinadas patologías, identificando grupos de riesgo temprano y orientándolos para la prevención.(Salles et al. 2018)

Existen múltiples opciones metodológicas en cuanto al equipo utilizado para capturar la imagen del iris: cámaras digitales, iridoscopios integrados y / o adaptados (cámaras específicas para fotografiar el iris), varios tipos de iluminación y grabación de imágenes, técnicas de creación de imágenes para su posterior software de análisis y edición de imágenes regular (p. ej., Corel Draw, Photoshop o programas específicos para imágenes de iris, como Iris 3D).(Fuentes, Barreto, and Bautista 2017) Durante la última década, las herramientas más nuevas y el conocimiento adicional han creado nuevas oportunidades para abordar la coherencia y la generalización entre los estudios. Una síntesis actualizada 
de la literatura actual puede considerarse un primer paso importante para lograr un consenso y desarrollar pautas que ayuden a los investigadores a tomar decisiones informadas con respecto a los diseños y métodos de investigación utilizados en Iridología.(Esteves et al. 2021)

Iridología significa el estudio del iris y el diagnóstico del iris. Es una ciencia que permite el conocimiento sobre física, aspectos emocionales y mentales del individuo a través del iris. La Iridología es uno de los cuidados complementarios prácticos (CHPs) basadas en la comprensión holística del ser humano.(Flower 2016) El enfoque holístico establece que las totalidades representan más que la suma de sus partes. Éstas pueden ser organismos biológicos, organizaciones, sociedades o complejos científicoteóricos.(Salles et al. 2018)

Órganos y estructuras representados en el iris: se han desarrollado dos diagramas diferentes para poder llegar a un diagnóstico mediante el iris, ya que, el diagnóstico no solo se basa en encontrar manchas concretas en el iris del ojo, sino que, es una tarea que conlleva mucho más trabajo.(Bartholomew and Likely 2016)

El primero se trata sobre anillos concéntricos donde el más cercano a la pupila refleja el estómago y el externo la piel.(Far 2019)

\section{Los siete anillos del iris (1999), según cita Rita M. Holl.(Rita 1999)}

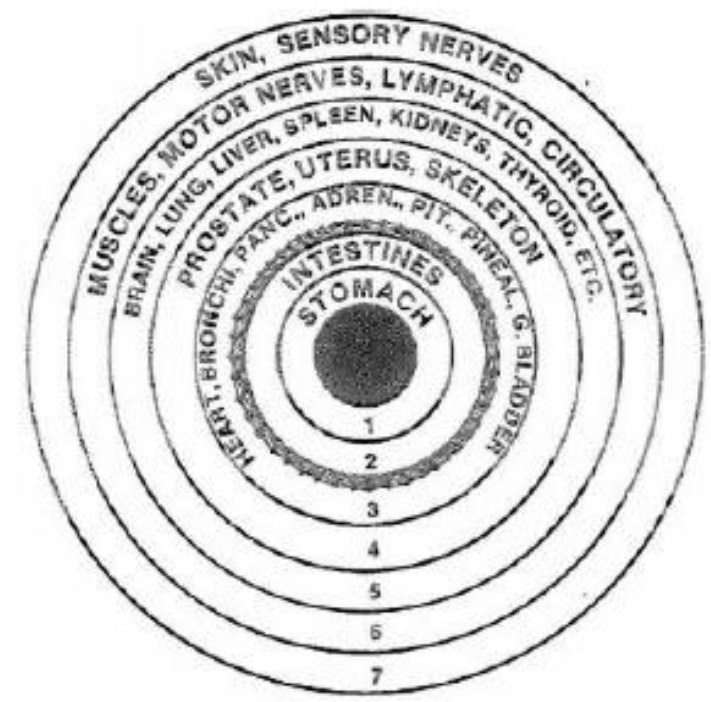

Al contrario, el segundo diagrama, este divide el iris en zonas radiales que se leen por zonas horarias. Por ejemplo, las dolencias del riñón se localizan entre las 6:00 h y las 7:00 h en la pupila izquierda. Se originan así entre 60 y 80 sectores.(Muzamil et al. 2020) 

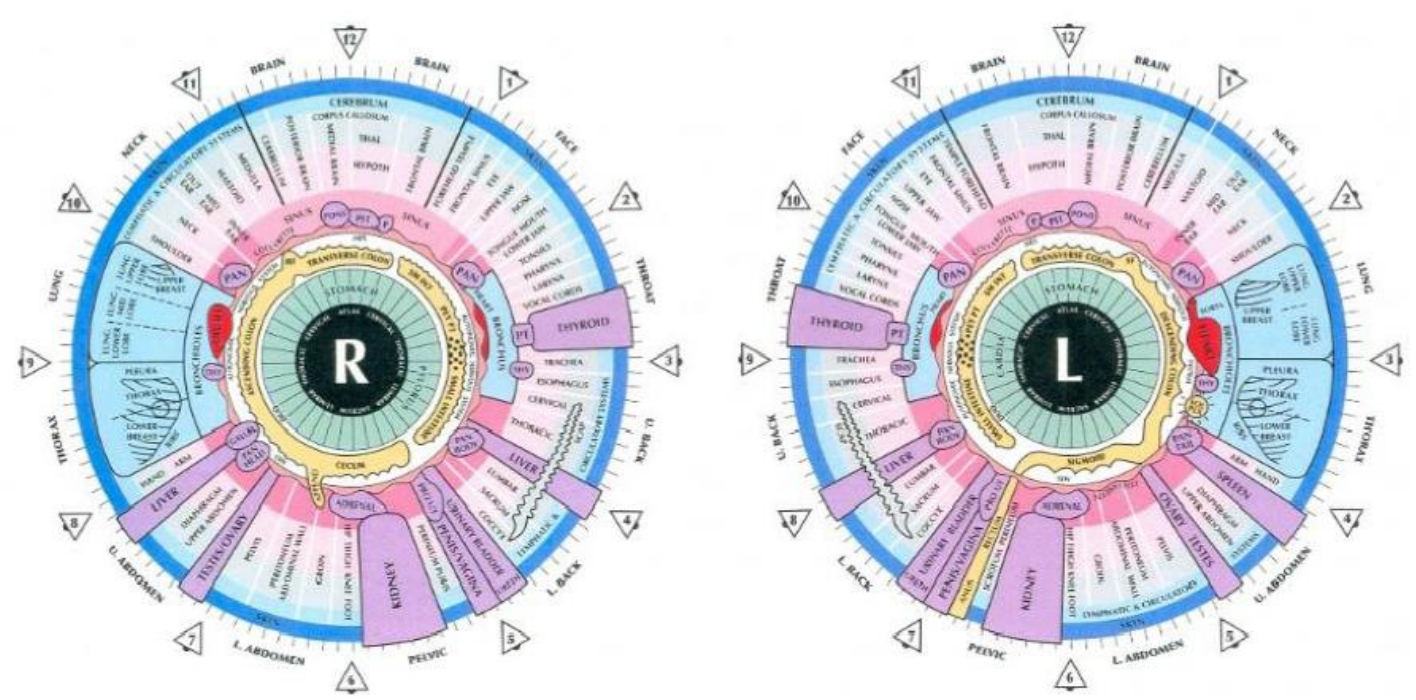

Carta Iridológica de sectores (2017), según cita Martiana et al.(Martiana et al. 2017)

Los órganos y estructuras que se localizan en la parte izquierda del cuerpo se representan en el iris izquierdo y viceversa. Sin embargo, los órganos y estructuras localizadas a ambos lados del cuerpo o en el centro de éste, se representan en ambos iris.(Llergo 2020)

A nivel de Latinoamérica, a mediados del siglo $\mathrm{XX}$, en varios países existió una expansión cultural, siendo relacionada con las nuevas enfermedades degenerativas que dio paso a la difusión de la Medicina alternativa, conformada por las tradiciones indígenas como; adivinadores, sobadores, yerberos, curanderos, chamanes y hueseros. Incluyendo también otras prácticas médicas, como son; la aromaterapia, la Iridología, la homeopatía, el naturismo y la terapia neura.(Jose Luis Herrera Lopez, Ayari Guadalupe Avila Larrea, Violeta Maricela Dalgo Flores, Evelyn Fernanda Velasco Acurio 2018)

En el Ecuador es justo reconocer que se han determinado nuevas políticas de estado en relación con la medicina tradicional y alternativa, toda esta "normativa legal" presume un respaldo para el desarrollo más visible de la medicina alternativa y ancestral en Ecuador y Riobamba, pero todavía se mantiene dentro de los límites de la información empírica en cuanto al conocimiento y aceptación de la población sobre su práctica.(Valarezo 2017) De tal manera que, la medicina alternativa es una práctica que se ha venido ejerciendo desde tiempos muy antiguos hasta la actualidad estando presente en todas las civilizaciones y culturas de la humanidad; ha evolucionado hasta incluirse en los sistemas de salud a nivel internacional, por ejemplo, Estados Unidos de Norteamérica, China, 
Brasil, en donde también podríamos mencionar al Ecuador, entre otros lugares.(Ingrid Roxana Ugalde Gruezo 2021)

Actualmente el estado ecuatoriano propone la construcción de un nuevo derecho ciudadano de buen vivir, en armonía con la naturaleza y con la persona misma, para proporcionar espacios de convivencia saludable y de bienestar, en función de lo cual ha establecido el Plan Nacional del Buen Vivir, dentro de las terapias alternativas más conocidas están: acupuntura y homeopatía, y las menos conocidas son: Reiki-sanación de manos e Iridología.(Valarezo 2017) 


\section{Cuadro de metaanálisis}

Práctica de la iridología como herramienta diagnóstica en medicina natural

\begin{tabular}{|c|c|c|c|}
\hline Nombre artículo & Resultados & Nombre artículo & Resultados \\
\hline $\begin{array}{l}\text { Determining diabetes } \\
\text { using iris recognition } \\
\text { system.(Bansal, } \\
\text { Agarwal, and Sharma } \\
\text { 2016) }\end{array}$ & $\begin{array}{l}\text { Los resultados obtenidos en esta investigación } \\
\text { son efectivos y alentadores, ya que, se ha } \\
\text { alcanzado una } \\
\text { precisión de un } 87,5 \% \text { en diagnósticos } \\
\text { del iris para diabetes.(Bansal et al. 2016) }\end{array}$ & $\begin{array}{l}\text { Controversia } \\
\text { sobre la } \\
\text { Iridología.(Llergo } \\
\text { 2020) }\end{array}$ & $\begin{array}{l}\text { Según Llergo,(Llergo 2020) afirma que se trata de una } \\
\text { pseudociencia sin valor diagnóstico, aunque los métodos de } \\
\text { algunos naturópatas pueden servir para promocionar la salud, mas } \\
\text { no para diagnosticar.(Llergo 2020) }\end{array}$ \\
\hline $\begin{array}{l}\text { Controversia sobre } \\
\text { la Iridología.(Llergo } \\
\text { 2020) }\end{array}$ & $\begin{array}{l}\text { En Alemania la Iridología es una técnica de } \\
\text { diagnóstico alternativa muy aceptada, se dice } \\
\text { que cerca del } 80 \% \text { de los Heilpractiker alemanes } \\
\text { (profesionales de la salud no calificados } \\
\text { médicamente) usan la iridología como principal } \\
\text { método para solucionar los problemas de salud } \\
\text { de sus pacientes.(Llergo 2020) }\end{array}$ & $\begin{array}{l}\text { Parameters to } \\
\text { increase the } \\
\quad \text { quality of } \\
\text { Iridology studies: } \\
\text { A scoping review. } \\
\text { (13) }\end{array}$ & $\begin{array}{l}\text { Según Esteves, Moreno, Pereira.,(Esteves et al. 2021) quien } \\
\text { vinculó la evaluación de la singularidad del individuo con la } \\
\text { promoción de la salud y como una estrategia para evitar problemas } \\
\text { de salud en la salud, pero no para tener un diagnóstico preciso } \\
\text { mediante el iris.(Esteves et al. 2021) }\end{array}$ \\
\hline $\begin{array}{l}\text { Intelligent Iris Based } \\
\text { Chronic Kidney } \\
\text { Identification } \\
\text { System.(Muzamil et al. } \\
\text { 2020) }\end{array}$ & $\begin{array}{l}\text { La clasificación de falsos negativos mediante el } \\
\text { diagnóstico del iris es en porcentajes bajos entre } \\
1,8 \text { y } 2 \% \text {. } \\
\text { Y obteniendo un diagnóstico de riñón sano y } \\
\text { riñón crónico en un } 95,4 \% \text { y } 98,2 \% \text { según } \\
\text { corresponde.(Muzamil et al. 2020) }\end{array}$ & $\begin{array}{l}\text { Subsidising } \\
\text { Australian } \\
\text { pseudoscience: } \\
\text { is iridology } \\
\text { complementary } \\
\text { medicine or witch } \\
\text { doctoring? }{ }^{(17)}\end{array}$ & $\begin{array}{l}\text { Bartholomew y Likely ,(Bartholomew and Likely 2016) probó a } \\
\text { varios iridólogos destacados a quienes se les permitió examinar } \\
\text { fotografías en color del iris en } 78 \text { sujetos, y la mitad sufría de } \\
\text { vesícula biliar enfermedad. No solo fallaron los exámenes de iris } \\
\text { en distinguir entre sujetos sanos y aquellos con dolencias de la } \\
\text { vesícula biliar, ni siquiera por unanimidad, acordar un solo } \\
\text { diagnóstico. Se ha probado las capacidades de diagnóstico de tres } \\
\text { iridólogos, que eran tan pobres que ni siquiera reconocieron la } \\
\text { fotografía de un ojo de cristal.(Bartholomew and Likely 2016) }\end{array}$ \\
\hline
\end{tabular}




\begin{tabular}{|c|c|}
\hline Nombre artículo & Resultados \\
\hline $\begin{array}{l}\text { Detección de enfermedades mediante Zonas } \\
\text { somatotópicas mediante la segmentación } \\
\text { polar del Iris.(Muzamil et al. 2020) }\end{array}$ & $\begin{array}{l}\text { Es importante destacar que la Iridología es una medicina alternativa; por lo que el presente sistema de } \\
\text { pre diagnóstico pude auxiliar en la validación de este método de detección de enfermedades.(Far 2019) }\end{array}$ \\
\hline $\begin{array}{l}\text { Iridología como herramienta semiológica en } \\
\text { homeopatía.(Luna 2016) }\end{array}$ & $\begin{array}{l}\text { Los signos del iris evolucionan de acuerdo a la evolución de la enfermedad, pudiendo mostrar en la } \\
\text { variación de los signos, el efecto de cualquier intervención terapéutica.(Luna 2016) }\end{array}$ \\
\hline $\begin{array}{l}\text { Análisis sobre la homeopatía como ciencia o } \\
\text { pseudociencia.(Ochoa Ortega 2018) }\end{array}$ & $\begin{array}{l}\text { La mayoría de los estudios revisados son favorables al uso de los medicamentos homeopáticos en el } \\
\text { tratamiento de diferentes enfermedades. Existen varias teorías que explican sus mecanismos de } \\
\text { acción.(Ochoa Ortega 2018) }\end{array}$ \\
\hline $\begin{array}{l}\text { Homeopathy and Periodontal } \\
\text { Treatment in Type II Diabetic Patients: } \\
\text { a 1-Year Randomized Clinical Trial.(Mourão } \\
\text { et al. 2019) }\end{array}$ & $\begin{array}{l}\text { La experiencia clínica sugiere que es eficaz, relativamente económico y tiene una alta satisfacción del } \\
\text { paciente y una baja incidencia de efectos secundarios.(Mourão et al. 2019) }\end{array}$ \\
\hline
\end{tabular}




\section{CONCLUSIÓN}

Para concluir, podemos ver que la Iridología es muy poco reconocida en América Latina sobre todo en Ecuador, sin embargo, es un método diagnóstico muy eficaz según las diferentes investigaciones realizadas, un método que nos ayuda, no solo a diagnosticar las diferentes enfermedades, sino, también a poder prevenir las mismas y así poder gozar de un mejor estado de salud. Por otro lado, la medicina natural o conocida también como medicina tradicional u homeopatía, es una medicina que ayuda a realizar tratamientos a base de plantas y todo cuanto a naturaleza se refiere, dicha medicina alrededor del mundo es muy reconocida no solo por su bajo costo económico sino también por los buenos resultados obtenidos. Siendo en Ecuador muy reconocida ya que este es un país multiétnico y pluricultural, por lo que contamos con varias terapias naturales según las diferentes culturas existentes en todas las regiones del Ecuador tanto Sierra, Costa, Amazonía, incluso la región Insular.

\section{LISTA DE REFERENCIAS}

Bansal, Atul, Ravinder Agarwal, and R. K. Sharma. 2016. "Determining Diabetes Using Iris Recognition System.” International Journal of Diabetes in Developing Countries 35(4):432-38. doi: 10.1007/s13410-015-0296-1.

Bartholomew, R. E., and M. Likely. 2016. "Subsidising Australian Pseudoscience: Is Iridology Complementary Medicine or Witch Doctoring?" Australian and New Zealand Journal of Public Health 22(1):163-64. doi: 10.1111/j.1467842X.1998.tb01164.x.

Delgado Rodríguez, Miguel, and Silvia Palma Pérez. 2016. “Aportaciones de La Revisión Sistemática y Del Metaanálisis a La Salud Pública.” Revista Española de Salud Pública 80(5):483-89. doi: 10.1590/s1135-57272006000500007.

Ernst, E. Md. PhD. FRCP. 2016. "Iridology.Pdf.” Arch Ophthalmol 118.

Esteves, Rafael Braga, Juceli Andrade Paiva Morero, Sandra de Souza Pereira, Karina Dal Sasso Mendes, Kathleen Mary Hegadoren, and Lucilene Cardoso. 2021. "Parameters to Increase the Quality of Iridology Studies: A Scoping Review."

European Journal of Integrative Medicine 43(May 2020):101311. doi: 10.1016/j.eujim.2021.101311.

Far, Eduardo Bustos. 2019. "Deteccion de Patologias Mediante Zonas Somatotopicas Mediante La Segmentacion Polar Del Iris.” Revista Internacional de Sistemas 
Coputacionales y Electronicos 1-51.

Flower, Cyril H. 2016. "Ikidtagnosis -4nd Its Relation to Optometry." The Autralasian Journal of Optometry (June).

Fuentes, Oscar, Aldrin Barreto, and Veronica Bautista. 2017. "Segmentación de Iris Con OpenCV En Android.” Pistas Educativas (112):1464-84.

Ingrid Roxana Ugalde Gruezo, Marilyn Vilma Maffare. 2021. "CONOCIMIENTO Y USO DE LA MEDICINA ALTERNATIVA EN EL 'BARRIO VOLUNTAD DE DIOS’ DEL CANTON ESMERALDAS.”

Jose Luis Herrera Lopez, Ayari Guadalupe Avila Larrea, Violeta Maricela Dalgo Flores, Evelyn Fernanda Velasco Acurio, Ximena Alejandra Moyano Lopez. 2018. "Medicina e Interculturalidad." Mediciencias UTA 4.

Llergo, Maria Jesus Cano. 2020. "Controversia Sobre La Iridología.” Trabajo de Fin de Grado.

López González, Idania, Minerva Pérez González, and Linet Artiles López. 2016. "La Homeopatía Como Modalidad Terapéutica En América: Un Recuento Histórico Necesario." Revista Educación Médica Del Centro 8(S1):74-86.

López Vantour, Ana Caridad, Karelia Quevedo Peillon, Maricel Marquez Filiu, and Ana Maria Quinzán Luna. 2017. "Efectividad Del Tratamiento Homeopático En Extracciones Complicadas o Laboriosas.” Medisan 21(10):3032-37.

Luna, Silvia Cadena. 2016. "IRIDOLOGÍA COMO HERRAMIENTA SEMIOLÓGICA EN." FUNDACIÓN UNIVERSITARIA LUIS G. PÁEZ.

Marín Martínez, F., J. Sánchez Meca, and J. A. López López. 2019. “El Metaanálisis En El Ámbito de Las Ciencias de La Salud: Una Metodología Imprescindible Para La Eficiente Acumulación Del Conocimiento.” Fisioterapia 31(3):107-14. doi: 10.1016/j.ft.2009.02.002.

Martiana, K. Entin, Ali Ridho Barakbah, S. Syarifa Akmilis, and Aditya Afgan Hermawan. 2017. "Application for Heart Abnormalities Detection through Iris." Proceedings - 2016 International Electronics Symposium, IES 2016 315-22. doi: 10.1109/ELECSYM.2016.7861024.

Mourão, Leila, Romeu Carillo, Sabrina Martins Linares, Antonio Canabarro, and Ricardo Guimarães Fischer. 2019. "Homeopathy and Periodontal Treatment in Type Ii Diabetic Patients: A 1-Year Randomized Clinical Trial.” Brazilian Dental Journal 
30(2):139-45. doi: 10.1590/0103-6440201902124.

Muzamil, Sohail, Tassadaq Hussain, Amna Haider, Umber Waraich, Umair Ashiq, and Eduard Ayguadé. 2020. “An Intelligent Iris Based Chronic Kidney Identification System.” Symmetry 12(12):1-14. doi: 10.3390/sym12122066.

Ochoa Ortega, Max Ramiro. 2018. “Análisis Sobre La Homeopatía Como Ciencia o Pseudociencia.” Archivo Médico de Camagüey 22(3):381-92.

Oliveira, Ivia Fonseca de, Bráulio Henrique B. Peluso, Filipe A. C. Freitas, and Marilene Cabral do Nascimento. 2018. "Homeopatia Na Graduação Médica: Trajetória Da Universidade Federal Fluminense." Revista Brasileira de Educação Médica 42(1):94-104. doi: 10.1590/1981-52712015v42n1rb20160097.

Rita, M. 1999. “Iridology : Another Look.” Alternative Health Practitioner 5:35-43.

Sacristán Rubio, Alberto, and José Ignacio Torres Jiménez. 2016. "Homeopatía, Una

Realidad Social y Asistencial." Atencion Primaria 47(7):469-70. doi: 10.1016/j.aprim.2015.02.003.

Salles, Léia Fortes, Maria Júlia Paes da Silva, and Eutália Aparecida Cândido de Araújo. 2018. "The Prevalence of Iridologic Signs in Individuals with Diabetes Mellitus." Acta Paulista de Enfermagem 21(3):474-80. doi: 10.1590/s010321002008000300015.

Salles, Léia Fortes, and Maria Júlia Paes de Silva. 2018. "Iridology: A Systematic Review." Revista Da Escola de Enfermagem 42(3):585-89. doi: 10.1590/S008062342008000300026.

Valarezo, Carlos. 2017. “Aceptación de La Medicina Alternativa En La Población Urbana de Riobamba-Ecuador." REE Unach 8(1):1-7.

Yuliet, Heidy, and Caicedo Fandiño. 2019. “Calidad de Vida Laboral En Trabajadores de La Salud En Países.” Signos 11:41-62. 